\title{
An Unexpected Precipitant of Delirium in a Patient with Developmental Delay
}

Sandra Rao MD, and Lawrence Jacobs

\author{
About the Authors: \\ Sandra Rao is Resident Physician at University of Toronto. Lawrence Jacobs is Associate Dean at the University of Windsor. \\ Correspondence to Sandra Rao: sandra.rao@wchospital.ca \\ Submitted: November 30, 2017. Accepted: February 3, 2018. Published: November 9, 2018. DOI: 10.22374/cjgim.v13i4.259
}

\begin{abstract}
Delirium is an acute change in mental status with a fluctuating course. It has numerous precipitating factors that can be classified using the DIMS-R framework, including structural changes and metabolic disturbances. Delirium is often under-recognized and numerous screening tools have been developed to aid diagnosis.
\end{abstract}

\section{RESUME}

Le délire est un changement aigu de l'état mental avec une évolution fluctuante. Il contient de nombreux facteurs précipitants pouvant être classés à l'aide du cadre DIMS-R, notamment des changements structurels et des perturbations métaboliques. Le délire est souvent méconnu et de nombreux outils de dépistage ont été développés pour faciliter le diagnostic.

\section{Case}

A 52-year-old female with a history of cerebral palsy was admitted to hospital for hypernatremia and a first episode of hyperosmolar hyperglycemic state. Upon resolution of her metabolic disturbances, she developed visual hallucinations which prompted further investigations revealing a surprising diagnosis.

\section{Conclusion}

Visual hallucinations are the most common psychotic feature associated with delirium and are more likely to be associated with multiple medical conditions. Delirium is often multi-factorial and a full diagnostic workup, including imaging studies, should be considered to address all possible underlying etiologies. Effective treatment of delirium is dependent on treatment of all of its precipitants.

\section{Case}

A 52-year-old female of French descent with cerebral palsy initially presented following a two-day history of weakness, polyuria, and confusion. She had a lifelong history of developmental delay and long-term memory loss, but could communicate with short sentences in French. At baseline, the patient ambulated at home independently with a cane.
At presentation, her temperature was $36.3^{\circ} \mathrm{C}$, pulse was regular at 76 beats per minute, blood pressure was 106/69, and oxygen saturation $98 \%$ on room air. She was noted to have macrocephaly, left-sided hemiparesis with decreased muscle bulk and atrophy, all of which existed at baseline. Her general exam was notable for signs of hypovolemia but was otherwise unremarkable. She was admitted with a first episode of hyperosmolar hyperglycemic 
state after investigative bloodwork, and was fluid-resuscitated and treated with regular insulin. The patient's initial bloodwork was also significant for hypernatremia at 167 , which was corrected with parenteral and oral hydration. She was later transitioned to oral anti-glycemic agents upon improvement of her hyperglycemia.

During her stay, the patient's metabolic disturbances and mental status stabilized initially, however she began having visual hallucinations the morning of her pending discharge.

A computed tomography (CT) scan of the head without contrast was then performed, revealing massive enlargement of the ventricular system. The lateral ventricles exhibited a marked degree of mass effect with very little remaining parenchyma. On the left side, there was a thin rim of persistent cortical and white matter, although due to the degree of hydrocephalus, it was thinned and displaced to under the surface of the calvarium. On the right side, there was essentially no tissue remaining within the vicinity of the frontal lobe with very little within the vicinity of the parietal lobe. There was also evidence for a low-density extra-axial fluid collection along the right frontal lobe, likely due to a chronic subdural hematoma or a hygroma. The findings were consistent with an extreme degree of hydrocephalus. The CT was followed by magnetic resonance imaging of the head; the results were consistent with aqueductal stenosis based on a normal sized fourth ventricle (Figure 1).

Neurosurgery was consulted following imaging studies. A third ventriculostomy was performed with the guidance of
BrainLAB and a programmable ventriculoperitoneal (VP) shunt was inserted. She recovered from this procedure with no acute intracranial hemorrhage, although no change in the marked enlargement of the ventricles was noted immediately postoperatively. Her level of alertness, however, did soon return to her baseline and her visual hallucinations resolved.

On her two-month follow-up, her hydrocephalus persisted with no notable change in her ventriculomegaly; but, according to her family, she was walking better, with less unsteadiness. She was no longer experiencing any psychotic symptoms.

\section{Discussion}

Delirium is a common presentation of medical illness with numerable precipitants. It has several hallmark features, including disturbances in cognition that develop over short periods of time - generally hours to days - that can be explained by a medical condition, substance use, or medication side effect. ${ }^{1}$ The DIMS- $\mathrm{R}^{2}$ criteria organizes the causes of delirium into groupings including drugs, infection, metabolic abnormalities, structural insults, and urinary or fecal retention. The cognitive features associated include inattention, disorientation, and memory changes. Features of psychosis and agitation may be present as well. ${ }^{2}$ Delirium has been found to be correlated with worse outcomes, but is often under-recognized. ${ }^{3}$ Approximately $60 \%$ of cases are misdiagnosed, missed, or detected late. To address this inconsistency, various delirium screening tools have been
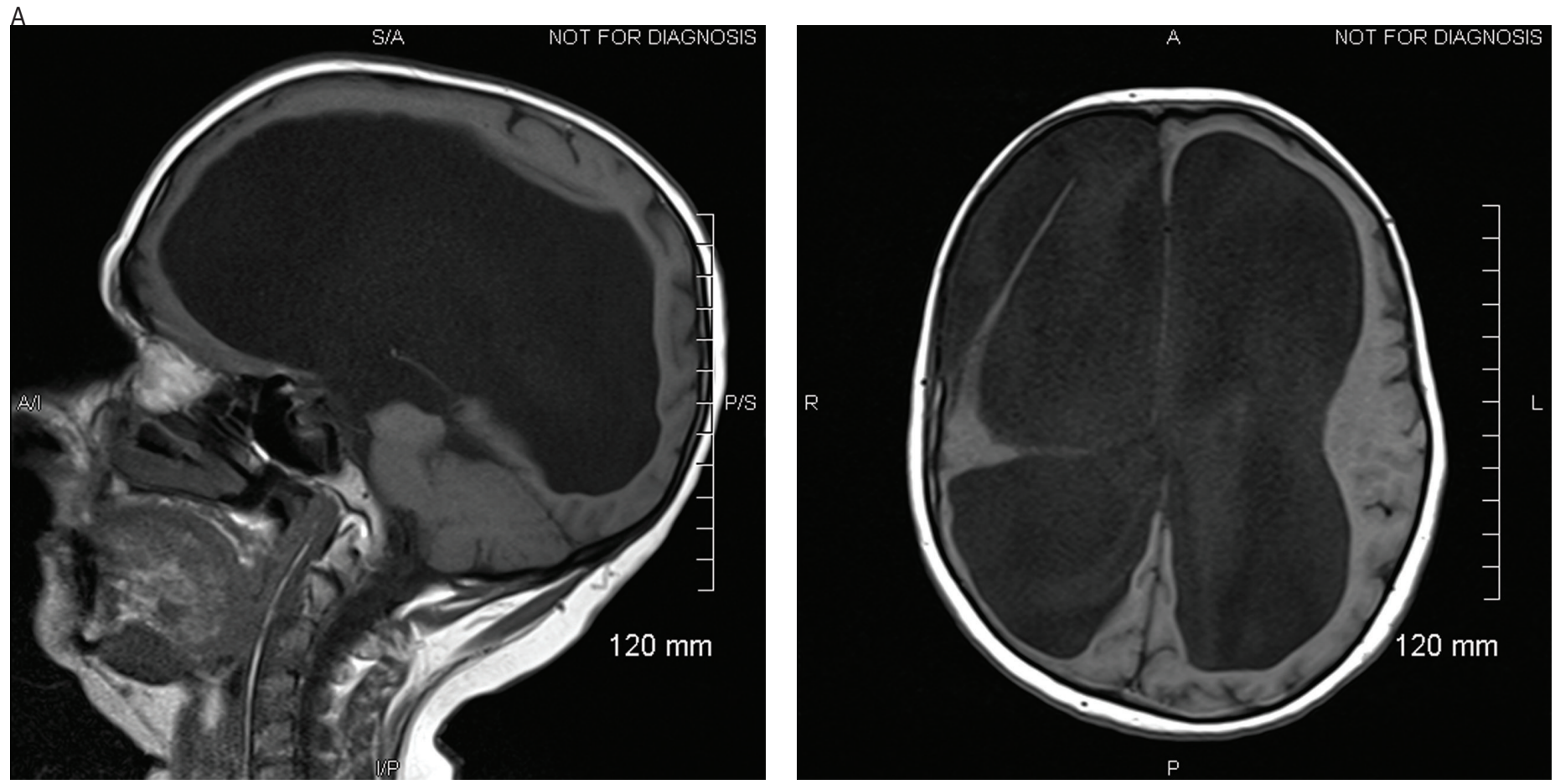

Figure 1. Magnetic resonance imaging demonstrating saggital (left) and axial (right) views of severe ventriculomegaly secondary to aqueductal stenosis. The mass effect of the cerebrospinal fluid resulted in significant loss and displacement of brain tissue. 
developed for the inpatient setting, including the 4 "A's" Test ${ }^{4}$ and the confusion assessment method (CAM) ${ }^{5}$ In this case report, we presented a patient who developed a multi-factorial cause of delirium.

Changes in cognitive function from baseline should prompt a full differential diagnosis. Following the DIMS-R framework may aid in the workup, since delirium is a clinical diagnosis that is generally not attributed to a single pathology. ${ }^{2}$ Imaging studies should be considered as part of the delirium workup to rule out structural changes. Treatment of delirium is only effective when all underlying causes are identified and addressed. Our patient had multiple possible causes of delirium. Metabolically, she presented with marked hypernatremia and hyperglycemia, which are both classic precipitants of delirium. Structurally, the patient's profound hydrocephalus may have also contributed, considering the clinical improvement she experienced following VP shunt insertion. The key feature of her presentation which prompted further workup of the patient's disturbance in cognition is that she developed unexplained visual hallucinations despite the correction of her metabolic abnormalities and initial resolution of her confusion. In the absence of infectious symptoms, this suggested possible underlying cerebral pathology.

Hallucinations can be the product of one or more of the following processes: organic brain disease, neurochemical changes, or psychodynamic forces. While visual hallucinations are a classical feature of psychosis, they are the most common psychotic manifestations of delirium, and are more likely to be associated with multiple medical disorders. ${ }^{6}$ Other common medical causes of visual hallucinations include dementia, migraines, and seizure activity. Vision impairment can also cause visual hallucinations, such as in Charles Bonnet syndrome and Anton's syndrome. ${ }^{7}$

The clinical presentation of hydrocephalus and other structural causes of delirium is highly variable and is related to the degree and nature of anatomical deformity present. The patient in this case was found to have severe ventriculomegaly consistent with Long-standing Overt Ventriculomegaly (LOVA), which was first described by Oi and his colleagues. Patients with LOVA have infantile hydrocephalus secondary to congenital aqueductal stenosis that slowly progresses into adulthood. In congenital hydrocephalus, the duration of the injury is another factor that determines the presentation of symptoms. LOVA presents as a triad of headaches, subnormal IQ, and macrocephaly. Other features such as gait disturbances, urinary incontinence, and severe depression may be present as well. ${ }^{8}$

In LOVA, there is marked progressive dilatation of ventricles and significant loss of cerebral parenchyma. This was seen in this patient, such that there was "virtually no brain." Interestingly, this patient does have significant functional abilities, demonstrating a remarkable discrepancy between her anatomy and clinical outcome. Our patient could ambulate with cane assistance and was able to hold short conversations with reasonable short-term memory despite having very minimal cerebral cortex for motor function and language processing. These findings are a significant demonstration of the role of neuroplasticity in preservation of cognitive and neurological function during infancy. It may also be suggestive of the redundancy present in the cerebral cortex, or an increased role of subcortical structures in higher processing. ${ }^{9}$ Another possible explanation for the degree of functional preservation is that hydrocephalus is a disease of white matter, but grey matter is relatively spared even in severe ventriculomegaly. ${ }^{10}$ Nevertheless, the patient's lack of anatomical improvement following VP shunt insertion sheds light on the therapeutic challenges in treating LOVA as the brain loses compliance with such a degree of hydrocephalus. Such a degree of craniocerebral disproportion increases the risk of subdural hematoma and low-ICP syndrome following shunt insertion. ${ }^{8}$

This case is reflects the various complexities in cognitive reserve. Despite the notable adaptive mechanisms of the brain seen in our patient's lifelong hydrocephalus, the onset of delirium also attests to the neurochemical vulnerability of her brain from her underlying cognitive impairment. As a consequence, identifying and addressing risk factors for delirium will be paramount in providing future medical care to improve clinical outcomes.

\section{Disclaimer}

This case report has never been published and has not been submitted to any other journal for consideration. Our affiliations lie with the Schulich School of Medicine \& Dentistry and Windsor Regional Hospital. Furthermore consent was obtained from the patient in question to share their case in a case report for academic and educational purposes.

\section{References}

1. American Psychiatric Association. Diagnostic and Statistical Manual of Mental Disorders. 5th ed. Arlington, VA: American Psychiatric Publishing; 2013.

2. Neufeld KJ, Thomas C. Delirium : definition, epidemiology, and diagnosis. J Clin Neurophysiol 2013;30(5):438-42.

3. Marcantonio E. Delirium severity and psychomotor types: their relationship with outcomes after hip fracture repair. J Am Geriatr Soc 2002;50(5):850-57.

4. Bellelli G, Morandi A, Davis DHJ, et al. Validation of the 4AT, a new instrument for rapid delirium screening: a study in 234 hospitalised older people. Age Ageing 2014;43(4):496-502. doi:10.1093/ageing/afu021.

5. Monette J, Galbaud Du Fort G, Fung SH, et al. Evaluation of the confusion assessment method (CAM) as a screening tool for delirium in the emergency room. Gen Hosp Psychiatr 2001;23(1):20-25. doi:10.1016/ S0163-8343(00)00116-X.

6. Webster R, Holroyd S. Prevalence of psychotic symptoms in delirium. Psychosomatics 2000;41(6):519-22. doi:10.1176/APPI.PSY.41.6.519. 
7. Teeple RC, Caplan JP, Stern TA. Visual hallucinations: differential diagnosis and treatment. Prim Care Companion J Clin Psychiatr 2009;11(1):26-32. Available at: http://www.ncbi.nlm.nih.gov/pubmed/19333408.

8. Oi S, Sato O, Matsumoto S. Neurological and medico-social problems of spina bifida patients in adolescence and adulthood. Child's Nerv Syst 1996;12(4):181-87. doi:10.1007/BF00301248.
9. Canu EDG, Magnano I, Paulus KS, et al. Neuropsychophysiological findings in a case of long-standing overt ventriculomegaly (LOVA). Neurosci Lett. 2005;385(1):24-29. doi:10.1016/J.NEULET.2005.05.026.

10. Lewin R. Is your brain really necessary? Science 1980;210(4475):1232-34. 